\title{
Neutralization Diversity of HIV-1 Indian Subtype C Envelopes Obtained From Cross Sectional and Followed up Individuals Against Broadly Neutralizing Monoclonal Antibodies Having Distinct Gp120 Specificities
}

Ranajoy Mullick

International AIDS Vaccine Initiative and Translational Health Science \& Technology Institute Jyoti Sutar International AIDS Vaccine Initiative and Translational Health Science \& Technology Institute Nitin Hingankar

Translational Health Science \&Technology Institute

\section{Suprit Deshpande}

Translational Health Science \& Technology Institute

Madhuri Thakar

National AIDS Research Institute

Seema Sahay

National AIDS Research Institute

Rajesh Ringe

IMTECH: Institute of Microbial Technology CSIR

\section{Sampurna Mukhopadhyay}

National AIDS Research Institute

Ajit Patil

National AIDS Research Institute

Shubhangi Bichare

National AIDS Research Institute

Kailapuri G Murugavel

YRG CARE: YR Gaitonde Centre for AIDS Research and Education

Aylur K Srikrishnan

YRG CARE: YR Gaitonde Centre for AIDS Research and Education

\section{Rajat Goyal}

International Aids Vaccine Initiative

\section{Devin Sok}

International Aids Vaccine Initiative 
Jayanta Bhattacharya ( $\sim$ JBhattacharya@iavi.org )

International AIDS Vaccine Initiative and Translational Health Science \& Technology Institute https://orcid.org/0000-0002-1531-3822

\section{Research}

Keywords: HIV-1, clade C, neutralizing antibodies, envelope, VRC01, CAP256-VRC26.25, PGDM1400, PGT121, India

Posted Date: January 19th, 2021

DOl: https://doi.org/10.21203/rs.3.rs-148517/v1

License: (c) (i) This work is licensed under a Creative Commons Attribution 4.0 International License. Read Full License 


\section{Abstract}

Background. The potential use of the broadly neutralizing monoclonal antibodies (bnAbs) towards prophylaxis and treatment to HIV-1 is currently being explored. While a number of promising bnAbs have been discovered and few of them have progressed towards clinical development, their extent of neutralization coverage with respect to global HIV-1 variants given the existence of genetically distinct subtypes and recombinants circulating globally is not clearly known. In the present study, we examined the variation in the neutralization susceptibility of pseudoviruses expressing 71 full length primary HIV-1 subtype $C$ envs obtained from limited cross-sectional individuals over different time points against four bnAbs that target gp120 with distinct specificities: VRC01, CAP256-VRC26.25, PGDM1400 and PGT121.

Results. We found significant variations in the susceptibility of Indian clade $\mathrm{C}$ to these four bnAbs and were found to be distinct to that observed with that of African subtype $\mathrm{C}$ based on the existing datasets and were also found to be concordant with their sequence diversity. Trend analysis indicated an increasing neutralization resistance observed overtime with CAP25-VRC26.25, PGDM1400 and PGT121 when tested on pseudoviruses expressing envs obtained from 1999-2016. Our data was found to be distinct from what was observed in case of African HIV-1 subtype C. However, inconsistent trend in neutralization susceptibility was observed, when pseudoviruses expressing envs obtained from three followed up individuals were examined. Finally, through predictive analysis of the 98 Indian subtype $C$ including those assessed in the present study by employing additive model implemented in CombiNAber (www.hiv.lanl.gov), we observed two possibilities where combinations of three bnAbs (VRC01/CAP56VRC26.25/PGT121 and PGDM1400/CAP256-VRC26.25/PGT121) could achieve near 100\% neutralization coverage.

Conclusions. Our findings not only indicate disparate intra-clade $\mathrm{C}$ genetic vis-à-vis neutralization diversities but also warrants the need for more comprehensive study using additional isolates towards comparing inter and intra-clade neutralization diversities which will be necessary for selecting the bnAb combinations suitable for optimal coverage of the region-specific HIV-1 circulating subtypes. Expanding these efforts is imperative for designing efficacious bnAb based intervention strategies for India as well as subtype $\mathrm{C}$ in general.

\section{Introduction}

The development of a preventive vaccine to HIV-1 with a potential to tackle the enormous genetic diversity remains a challenge. This genetic variation is primarily accounted by the HIV-1 envelope gene (env) which is responsible for greater than $35 \%$ difference in amino acid sequences between different subtypes [1]. A number of broadly neutralizing monoclonal antibodies (bnAbs) capable of cross neutralizing diverse genetic subtypes have been discovered from elite neutralizers since 2009, which have fuelled, in recent times their potential use in prevention and therapy over and above the existing antiretroviral (ARV) drugs [2-9]. Few of these bnAbs have also advanced into clinical development based on their promising antiviral activity observed in animals and human clinical trials [10-16]. In general, 
breadth and potency of bnAbs are defined by their extent in neutralizing a panel of pseudoviruses expressing envs derived from various subtypes and recombinants representing different geographic regions. Hence, the suitability of bnAbs that exhibited highest breadth to overcome region-specific diversity is not clearly known. Previous studies have shown that although bnAbs isolated from individuals infected with one particular subtype are generally effective at neutralizing viruses belonging to other subtypes, antibody potency is often found to be correlated with matched subtypes [17-21]. Moreover, viral and host diversities have been found to have an impact even within matched subtype, as demonstrated by the fact that the subtype-matched neutralization advantage was more apparent in regions with distinct viral diversities $[19,22,23]$.

HIV-1 subtype $\mathrm{C}$ accounts for approximately half of the global infections [24], which predominates in India and South Africa. Recently very few studies have attempted to understand a few selected bnAbs for their extent to neutralize HIV-1 subtype $C$ of African origin either singly and/or in combinations [25-27], however those studies did not include pseudoviruses expressing envs representing Indian subtype $\mathrm{C}$. It is possible that an intra-clade $\mathrm{C}$ specific neutralization patterns may exist. For example, Rademeyer et al. [28] have shown that subtype $C$ viruses of African origin have become more resistant to VRC01, PG9 and 4E10 compared to CAP256-VRC26.25 and PGT128. In the present study, we examined the variation in neutralization sensitivity of pseudoviruses expressing 71 complete HIV-1 subtype $C$ primary envs collected over a period of time (1999-2014) against four bNAbs (VRC01, CAP256-VRC26-25, PGDM1400 and PGT121) that are not only most broad and potent amongst the best bnAbs discovered with distinct epitope specificities on viral envelope to date but also amongst ones that are farthest in under clinical development and testing [29-31].

\section{Results}

\section{Evidence of variation in susceptibilities of HIV-1 India clade C to bnAbs targeting gp120 having distinct target epitope specificities.}

We first examined the degree of neutralization sensitivity of HIV-1 Indian clade $\mathrm{C}$ to four bnAbs: VRC01 (having CD4-binding site specificity), PGDM1400 and CAP256-VRC26.25 (both having V1V2 specificity) and PGT121 (target V3 supersite). A total of 71 pseudoviruses expressing complete env gene ( $g p 160)$ obtained from 40 disease stage specific samples from 28 HIV-1 infected individuals from Southern and Western India during the years 1999-2014 (the complete details of the these env clones along with their genetic properties are given in Table S1) were assessed for their degree of neutralization sensitivity towards four selected bNAbs of distinct specificities in TZM-bl neutralization assay. Several of these envs have been reported earlier [32-38]. As shown in Table 1, the degree of susceptibility of Env-pseudotyped viruses to the bnAbs varied. The neutralization data demonstrated that while VRC01 exhibited most breadth (neutralized 62/71 viruses), PGDM1400 exhibited least (46/71) of all the four bnAbs assessed. The neutralization coverage was assessed by measuring the ability of each of the bnAb to demonstrate 
$50 \%$ virus neutralization by $\mathrm{IgG}$ concentration less than or equal to $5 \mu \mathrm{g} / \mathrm{ml}\left(\mathrm{IC}_{50}\right)$. In addition, CAP256VRC26.25 was found to neutralize the virus panel with maximal potency $\left(\mathrm{IC}_{50}\right.$ of 0.0033 with $76 \%$ breadth). Interestingly, while neutralization resistance of Env-pseudotyped viruses to all four bnAbs was found to be associated with known resistance signatures (Table S2), for few envs, no known resistance signatures were evident, suggesting alternate mechanism of neutralization resistance. Overall, our data showed variation in neutralization breadth and potency of VRC01, CAP256-VRC26.25, PGDM1400 and PGT121 to HIV-1 Indian clade C envelopes that were tested in this study.

\section{Comparing neutralization diversity of Indian clade $\mathrm{C}$ with other globally circulating subtypes and recombinants.}

We next examined whether inter and intra clade env diversity have any association with altered neutralization phenotype. For this first we retrieved full length gp 160 sequences of Env-pseudotyped viruses reported in the CATNAP database (www.hiv.lanl.gov) and compared diversity by building a phylogenetic tree (Fig. 1A). As expected, our data very clearly demarcated distinctness of Indian clade $\mathrm{C}$ with non-Indian clade $\mathrm{C}$ and other subtypes (Fig. 1A; top left). This observation also corroborates with our earlier finding [39]. The neutralization scores (IC 50 values) against VRC01, CAP256-VRC26.25, PGDM1400 and PGT121 of most of the same retrieved gp160 sequences of all subtypes were next obtained from the CATNAP database for the purpose of comparing with that obtained in the current study. A total of 1020 gp160 sequences were used for the analysis with the following distribution: Subtype C (Pan Africa):290, Subtype C (India): 98 that includes 71 used in the present study, Subtype A: 76, Subtype B: 255, Subtype D: 42, CRF01_AE: 70, CRF07_B/C recombinants: 49, other subtypes and recombinant forms: 140 were assessed for their phylogenetic relatedness using IQtree (HIVdb model, non-parametric 1000 fast bootstrap with aSH-LRT test). Four heatmaps based on their responses to VRC01, CAP256-VRC26.25, PGDM1400 and PGT121 were built based on the phylogenetic tree to reflect the clustering based on their $\mathrm{IC}_{50}$ values obtained against each of these Env-pseudotyped viruses. As shown in Fig. 1A, We observed that while VRC01 appeared to be most broad, it was found to be least potent amongst all. CAP256VRC26.25 appeared to be most potent, however our data also indicates that it has been mostly assessed against HIV-1 subtype $C$ (including one used in the present study). These qualitative observations also reflected upon statistical assessment of viruses from India as indicated in Fig. 1B.

Finally, we compared the overall neutralization sensitivity against these four bnAbs of year matched (1999-2011), randomly selected equal number of viral gp160 datasets $(n=85)$ from CATNAP database with the ones assessed in this current study. The $\mathrm{IC}_{50}$ value of $5 \mathrm{ug} / \mathrm{ml}$ was considered as a threshold of neutralization sensitivity. As shown in Fig. 1C, the neutralization susceptibility of Indian clade $C$ envelopes to VRC01 were found to be distinct to that observed with the African clade $\mathrm{C}$ as well as other subtypes. Interestingly, while the degree of CAP256-VRC26.25 susceptibility between Indian clade C and other subtypes were observed to be comparable, this was found to be significantly different from African 


\section{Trend analysis of variation in neutralization sensitivity over time at population and individual levels}

We next examined the variation in neutralization sensitivity of HIV-1 env sequences sampled over time to these four bnAbs. For this, we grouped $\mathrm{IC}_{50}$ values (in three clusters) based on corresponding year of sampling (1990-2000, 2001-2010 and 2011-2016) of the viral env sequences. As shown in Fig. 2, the PGDM1400 sensitivity of Env-pseudotyped viruses obtained from both Indian and African population (Figure S1) was observed to be decreasing overtime. For PGT121 and VRC01 bnAbs, similar trend was observed in Indian population. When compared, a gradual increase in resistant neutralization phenotype of African subtypes to PGT121 and VRC01 was also observed overtime, it was, however, not found to be significant (Figure S1). Interestingly, an increasing trend in neutralization resistance of Indian clade C envelopes to CAP256-VRC26.25 was observed in contrast to that with envelopes obtained from African population (Figure S1). Overall, we observe that although number of Indian clade C envelopes tested in this study were not large enough for an absolute conclusion, our data clearly indicate differences in neutralization susceptibility trend against these four bnAbs overtime between Indian clade $C$ and other subtypes.

We next examined the trend of neutralization susceptibility of pseudoviruses expressing primary HIV-1 clade $C$ envs obtained from three individuals at different time points to VRC01, CAP256-VRC26.25, PGDM1400 and PGT121. Several of these envs were reported earlier by our group [35]. The primary envs (complete gp 160 sequences) from the donor NARI IVC-2 were obtained at following time point: between $0-6,12,18,24,36$ and 57 months; from donors NARI IVC-3 and NARI IVC-4 at 0-6, 12 and 18 months respectively. As demonstrated in Fig. 3 , the trend of susceptibility of pseudoviruses expressing envs isolated from these three donors overtime to VRC01, CAP256-VRC26.25, PGDM1400 and PGT121 varied. Out of the three donors, in one (NARI IVC-11), a clear trend of increasing susceptibility of Envpseudotyped viruses to all the four bnAbs were observed. When tested against PGT121, for NARI IVC-2, pseudoviruses expressing envs obtained from baseline to two years, were found to be resistant, while pseudoviruses expressing envs obtained from subsequent time points till 57 months were found to be highly sensitive with $\mathrm{IC}_{50}$ over $0.01 \mathrm{ug} / \mathrm{ml}$. For all the resistant envs, N332 glycan residue was found to be absent. Interestingly, we observed that the env obtained from 57-month time point was sensitive to PGT121 despite lacking the N332 glycan. While this could possibly be compensated by the presence of a glycan at the 334 position as was reported for PGT128 with similar specificity [40], four pseudoviruses expressing envs isolated from NARI IVC-2 were found to be resistant to PGT121 despite containing N334. While pseudoviruses expressing envs obtained from all time points from this donor were found to be highly sensitive to CAP256-VRC26.25 with $\mathrm{IC}_{50}<0.001 \mathrm{ug} / \mathrm{ml}$, no increasing or decreasing trend in susceptibility to VRC01 and PGDM1400 of all the envelopes were observed. For the third donor, NARI IVC- 
3, envelopes obtained from all the time points were found to be resistant to V1V2 -specific bnAbs CAP256-VRC26.25 and PGDM1400, while they were found to be sensitive to VRC01 and PGT121, however no clear trend in neutralization sensitivity over time observed. While resistance to different

bnAbs was found to be associated with absence of known target motifs, we found evidence of neutralization resistance in presence of known epitopes that are targets of some bnAbs (Table S2). Overall, we found that while increasing neutralization resistance trend was observed at the population level, when cross sectional virus isolates across years were analyzed such a trend was not evident with longitudinal viral envs obtained from three followed up Indian donors.

\section{Predicting optimal bnAb combination using experimental data.}

Finally, based on the neutralization data of all the Indian clade $C$ envelopes assessed in this study and neutralization scores of additional Indian clade $\mathrm{C}$ envs retrieved from CATNAP database (www.hiv.lanl.gov), we attempted to predict the optimal combination amongst these four bnAbs that would likely confer maximal neutralization coverage with highest potency. Towards achieving this, we employed additive model implemented in CombiNAber tool (https://www.hiv.lanl.gov/content/sequence/COMBINABER/combinaber.html) to all possible combinations of these four bnAbs (VRC01, CAP256-VRC26.25, PGDM1400 and PGT121). A total of 98 Indian clade $C$ envelopes ( 71 from the current study and existing neuralization data of 27 additional Indian clade $\mathrm{C}$ retrieved from HIV database) for preparing for coverage analysis. As shown in Fig. 4A, the cumulative coverage analysis indicated that each of VRC01, CAP256-VRC26.25, PGDM1400 and PGT121 bnAbs individually could provide $80 \%, 70 \%, 65 \%$ and $74 \%$ coverage respectively at IgG concentration of equal to or below $5 \mu \mathrm{g} / \mathrm{ml}$. When assessed for the minimum combination that would provide maximal neutralization coverage, two combinations of three bnAbs each (VRC01 + CAP56-VRC26.25 + PGT121 and PGDM1400 + CAP256-VRC26.25 + PGT121) appear to provide 100\% cumulative coverage at total IgG concentration of $5 \mu \mathrm{g} / \mathrm{ml}$ or less as predicted by CombiNAber. In parallel, we also examined whether these two bnAb combinations of three bnAbs could achieve the maximal neutralization coverage at low doses. Towards this, the median $\mathrm{IC}_{50}$ values of Indian clade $C$ envelopes $(n=98)$ obtained against single and different combinations of all the four bnAbs were obtained and the coverage versus potency assessment was statistically validated by Mann-Whitney test on the dataset obtained by implementation of additive model by the CombiNAber tool with target limit of IgG concentration of $5 \mu \mathrm{g} / \mathrm{ml}$. As shown in Fig. 4B, we observed that combination of CAP256-VRC26.25 + PGDM1400 and PGT121 could achieve near 100\% breadth with highest potency (Fig. 4B). This was found to be strikingly different with what we observed with African subtype $C$, where two combinations of each of these four bnAbs appeared to provide near $100 \%$ neutralization coverage with maximal potency. For all the different combination we assessed, we compared the median $\mathrm{IC}_{50}$ obtained in our study with that reported for African clade $\mathrm{C}$ envelopes $(\mathrm{n}=$ 250) by retrieving neutralization $I C_{50}$ values from CATNAP database (www.hiv.lanl.gov). As expected, we found that the same combinations of three bnAbs (VRC01 + CAP256-VRC26.25 + PGT121 and 
PGDM1400 + CAP256-VRC26.25 + PGT121) demonstrated maximal coverage with geometric cumulative mean $\mathrm{IC}_{50}$ of 0.0109 and $0.01137 \mu \mathrm{g} / \mathrm{ml}$ respectively and are observed to be comparable to what was observed with combination of all four bnAbs. Of interest, when compared with African clade $C$ envelopes, we observed that combination of two bnAbs that commonly include CAP256-VRC26.25 (such as CAP256VRC26.25 + PGDM1400, CAP256-VRC26.25 + VRC01 and CAP256-VRC26.25 + PGT121) appear to provide comparable coverage with significantly lower doses than what we observed with the three bnAb combinations with Indian clade C viruses (Fig. 4B). This is likely because CAP256-VRC26.25 was found to demonstrate better neutralization coverage of African clade $C$ with increased potency compared to what we observed with Indian clade C viruses.

\section{Discussion}

Understudied and unexplored population and clade specific HIV-1 sequence diversity presents a formidable challenge that is likely to play a major hindrance in designing of effective antibody mediated intervention strategies. Subtype $\mathrm{C}$ being the most predominant subtypes circulating majorly in Africa and India, a greater understanding of the antigenic and neutralization diversity of HIV-1 subtype $\mathrm{C}$ env would facilitate understanding of optimal bnAb combination that could potentially overcome region-specific the intra-clade $\mathrm{C}$ diversity. Through this preliminary exploratory study, we attempted to elucidate the distinct neutralization phenotype of primary HIV-1 subtype $\mathrm{C}$ obtained from Indian patients to four key bnAbs that not only have demonstrated considerable breadth but also amongst those which are farthest into clinical development and testing. When analyzed against pseudoviruses expressing 71 primary envs, we observed that while VRC01 demonstrated maximum breadth, PGDM1400 showed the least amongst the four. Conversely, CAP256-VRC26.25 was found to neutralize over $76 \%$ viruses with maximal potency amongst others. Interestingly, while the neutralization resistance of the viruses to PGDM1400 while majorly found associated with known substitutions as found in HIV database (www.hiv.lanl.gov), for some resistant envelopes, no known resistant motifs were found, indicating mechanism plausible underlying unknown associated with PGDM1400 resistance. Interestingly, while CAP256-VRC26.25 and PGDM1400 target epitopes on the V1V2 loop, Indian clade $C$ envelopes were found to be more susceptible to CAP256-VRC26.25 with fewer resistant signatures compared to PGDM1400. This is an interesting and perhaps important observation which warrants the need to screen for additional significantly large population-based study to understand the frequency of occurrence of signature motifs and/or substitutions associated with neutralization resistance. This is particularly important while choosing bnAbs targeting V1V2 region for clinical use as V1V2 region represents the most hypervariable region and subtle changes in loop length, glycosylation patterns and substitutions will compromise with the antibody mediated virus neutralization efficacy [41]. Indeed, a significant difference in neutralization breadth and potency was observed when we compared the sensitivity of Indian subtype $C$ with that of African subtype $C$ based on the existing data. Interestingly though, while comparable sensitivity to PGDM1400 was observed between Indian and African subtype C, significant differences was observed 
when sensitivities to VRC01, CAP256-VRC26.25 and PGT121 were assessed. This observation further highlights the variation in population and geography based env gene diversity associated with neutralization diversities.

Trend analysis at the population level, with limited data sets indicated a sense of increasing resistance to all the four bnAbs tested in this study with envelopes isolated from Indian patients between the years 2011-2016 increasingly resistant to PGDM1400. This could possibly be linked to the differences in host characteristics and varied genetic bottlenecks which contribute to altered virus and antibody evolution pathways in the course of natural infection. Our observation while found reasonably comparable to what was observed with African subtype C, except for CAP256-VRC26.25, we noted that most if not all the African $\mathrm{C}$ envelopes were obtained from acute/early infection. In this line, given this was an observational study, we also hypothesize that the stage of the disease, origin of the env, and possible the geographic differences (within and outside India) could potentially influence on the neutralization phenotypes to draw any robust conclusion. At the individual level, while an increasing susceptibility trend was observed with PGT121, no consistent trend observed with other three bnAbs. While we tested envs obtained from follow up visits of only three individuals, it would be important to study envs obtained from more number of individuals in the same follow up setting. Such exercise would possibly help understand optimal combination of bnAbs that would be suitable for prophylaxis or early treatment versus the combination that would be more suitable to treat at the late disease stage.

One of the approaches that is widely been considered to provide maximal neutralization breadth at low dose and overcoming virus escape (particularly in the context of antibody mediated treatment) is a combination or additive approach of bnAbs having distinct epitope specificities. Based on our experimental data, we observed that combination of two V1V2 directed (CAP256-VRC26.25, PGDM1400) and one V3 supersite-directed (PGT121) bnAbs could achieve near 100\% neutralization coverage with maximal potency. Intriguingly, this observation was found to be distinct to what we observed with that of African subtype $C$ where combinations of two bnAbs could possibly provide near $100 \%$ neutralization coverage at highest potencies. Thus, our observation further indicate neutralization diversity between Indian and African subtype $\mathrm{C}$, It is to be noted that while majority of the African clade $\mathrm{C}$ env sequences analyzed in this study were derived from acute and early infection cases (www.hiv.lan.gov), majority of the Indian subtype $C$ env sequences used in the present study were obtained from individuals post 6 months of infection and at late disease stages. Assessment of late disease stages from both regions would be important to design bnAb based therapeutic strategies. While not much Indian subtype $\mathrm{C}$ env sequences from acute/early infection are available, we believe that it will be very important to obtain such sequences that will be necessary to define, select and optimize bnAbs and their combination for developing antibody mediated prevention strategies. Several factors might account for this variability. As a result of the continuous drift of HIV- 1 genome and epitopes, it is possible that divergent subtypes follow separate evolution pathways that may impact their degree of susceptibility to different bnAbs. This may also be due to the population-specific diverse and distinct selection pressure. Our hypothesis is supported by the fact that different classes of bnAbs isolated from HIV-1 infected individuals were influenced by both the infecting subtypes [23] and also by the ethnic origin [24][42]. In addition, the possibility of 
increasing resistance to bnAbs as observed $[25,26]$ in recent studies over the course of the epidemic warrants the necessity for continued surveillance of virus evolution that would impact ability of the promising bnAbs mediated neutralization coverage of region-specific HIV-1 diversities.

\section{Conclusion}

In conclusion, our data indicate disparate neutralization diversities of HIV-1 subtype $\mathrm{C}$ when assessed against four bnAbs with distinct epitope specificities and those that that are amongst the furthest into the clinical development and testing. This observation necessitates the urgent need to compare large number of env sequences across different geographies, risk groups and key populations to understand how disparate intra-clade genetic diversity could potentially impact on virus neutralization. This will inform choice of selection of the bnAb combinations that would be suitable for optimal coverage of the regionspecific HIV-1 circulating subtypes for prevention and treatment over and above the existing antiretroviral drugs.

\section{Methods}

\section{Ethics statement.}

The plasma and PBMC samples used for amplification of complete env genes by PCR were collected following respective institutional ethical approvals.

\section{Plasmids, antibodies and cell lines.}

Plasmid DNA encoding HIV-1 gp160 sequences published earlier are listed in Table 1. Plasmid DNA encoding heavy and light variable IgG sequences of the following monoclonal antibodies: PGT121, PGDM1400 and VRC01 were obtained from the IAVI neutralizing antibody center at the Scripps Research and CAP256.VRC26.25 was kindly provided by Prof Lynn Morris, National Institute of Communicable Diseases (NICD), Johannesburg, South Africa under an ongoing collaborative project. TZM-bl cells were obtained from the NIH AIDS Research Program. HEK-293T cell line was obtained from American Type Culture Collection (ATCC).

\section{Amplification and cloning of gp160.}

Full length env (gp160) genes were PCR amplified from HIV+ plasma samples with slight modification as described previously [37]. Briefly, viral RNA was extracted using a high-pure viral RNA kit (Roche Inc.) by following manufacturer's protocol, and cDNA was prepared by reverse transcription-PCR (RT-PCR) using a Superscript III first-strand synthesis kit (Invitrogen Inc.). Primer used for CDNA synthesis was EnvR1 5'GCACTCAAGGCAAGCTTTATTGAGGCTT -3' (HXB2: 9605-9632). Rev-Env gp160 cassette were amplified from the cDNA product using La Taq high fidelity DNA polymerase in the $1^{\text {st }}$ round (Takara Bio Inc.) and 
PrimeSTAR GXL high fidelity DNA polymerase (Takara Bio Inc.) in the second round. The primers used for the $1^{\text {st }}$ round was EnvF1: 5'- AGARGAYAGATGGAACAAGCCCCAG-3' (HXB2: 5550-5574) and EnvRP2: 5'GTGTGTAGTTCTGCCAATCAGGGAA -3' (HXB2: 9157-9181) while for the second round was Env IF: 5'CACCGGCTTAGGCATCTCCTATGGCAGGAAGAA -3' (HXB2: 5950-5982) and Env IR: 5'-

TATCGGTACCAGTCTTGAGACGCTGCTCCTACTC -3' (HXB2: 8882-8915). PCR condition followed in the first round was initial denaturation of $94 \llbracket \mathrm{C}$ for 2 mins followed by 12 cycles of $94 \rrbracket \mathrm{C}$ for $10 \mathrm{sec}, 60 \rrbracket \mathrm{C}$ for $30 \mathrm{sec}, 68 \rrbracket \mathrm{C}$ for $3 \mathrm{~min}, 23 \mathrm{cycles}$ of $94 \rrbracket \mathrm{C}$ for $10 \mathrm{sec}, 55 \rrbracket \mathrm{C}$ for $30 \mathrm{sec}, 68 \rrbracket \mathrm{C}$ for $3 \mathrm{~min}$ with final extension of

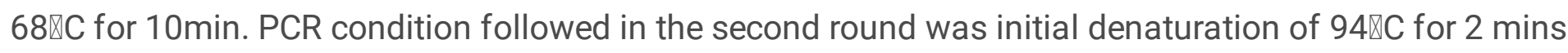
followed by 12 cycles of $94 \rrbracket C$ for $10 \mathrm{sec}, 62 \varangle \mathrm{C}$ for $30 \mathrm{sec}, 68 \otimes \mathrm{C}$ for $3 \mathrm{~min}, 23 \mathrm{cycles}$ of $94 \otimes \mathrm{C}$ for $10 \mathrm{sec}$, $60 \otimes \mathrm{C}$ for $30 \mathrm{sec}, 68 \otimes \mathrm{C}$ for $3 \mathrm{~min}$ with final extension of $68 \otimes \mathrm{C}$ for $10 \mathrm{~min}$. The gp 160 amplicons were purified and ligated into pcDNA 3.1/V5-His-TOPO (Invitrogen Inc.) vector or pSVIII as described before [35].

\section{Sequence analysis}

The gp160 amino acid sequences generated in the present study, those published in the GenBank earlier as well as those derived from the CATNAP database (supplementary information) were aligned to each other using Muscle v3.8.1551. The resulting alignment was curated using BioEdit sequence alignment editor v7.2.5 [43]. Variable loop regions for V1 (131-157: HXB2 numbering), V2 (158-196), V3 (296-331), V4 (386-417) and V5 (460-469) were retrieved from amino acid alignments with Extractalign implementation of the Emboss package [44]. Each of the loop datasets were then processed with custom bash/awk scripts to generate length statistics. Potential $\mathrm{N}$ linked glycosylation sites were predicted using $\mathrm{N}$-Glycosite tool available at the LANL HIV database [45]. Statistics regarding the antibody resistance associated signature residues were generated as described recently [39]. A phylogenetic tree was generated for all the HIV-1 viral clone sequences available in the CATNAP database along with those generated in the present study (Total $N=1020$ ) with iqtree under ' $H I V b$ ' model with estimated $\triangle$ parameters and number of invariable sites [46]. Robustness of the tree topology was further assessed by $\mathrm{SH}$-aLRT as well as 1000 ultrafast bootstrap replicates implemented in iqtree. The tree as well as neutralization IC50 ( $\mu \mathrm{g} / \mathrm{ML})$ values for each of the sequences against bnAbs PGDM1400, PGT121, VRC01 and CAP256-VRC26.25 were plotted with 'ggtree' package in R [47, 48]. IC50 values for year matched randomly selected equal number $(\mathrm{N}=85)$ were compared for HIV-1 clade $\mathrm{C}$ viral clones reported from India (including this study), Africa as well as other subtypes using Mann-Whitney test. Trend analysis for change in viral sensitivity to bnAbs VRC01, PGDM1400, CAP256-VRC26.25 and PGT121 was further performed. To achieve this, IC50 values for all Indian viral clones were grouped into three-time periods as per their year of sampling (1990-2000, 2001-2010 and 2011-2016) and compared with Jonckheere Terpstra test (JTT). CombiNAber (www.hiv.lanl.gov) [25], was used to predict optimal antibody combination for maximum neutralization of viral clones from India $(\mathrm{N}=98)$ and from Africa $(\mathrm{N}$ 250) using their experimentally determined IC50 values. Target IgG concentration of $5 \mathrm{ug} / \mathrm{mL}$ was considered as a threshold followed by implementation of the additive model in the CombiNAber tool to 
obtain geometric mean $\mathrm{IC}_{50}$ values for each combination. Sensitivity to each of these bnAbs combinations was further compared between Indian and African viruses using Mann-Whitney test. statistical computing software (v3.4.0) and R studio v1.0.143 [49, 50]. Statistical analysis for neutralization breadth and potency were done using GraphPad Prism version 5.01 for Windows, GraphPad Software, San Diego California USA. Scatter plots for bnAb potency distribution across geographically distinct populations as well as those from longitudinally collected samples were assessed statistically and plotted in GraphPad Prism v5.01.

\section{Preparation of Env-pseudoviruses}

Pseudotyped viruses were prepared by the co transfection of env-expressing plasmid DNA along with the plasmid DNA expressing HIV-1 genes with premature stop codon for env (pSG3DEnv) into 293T cells in 6well tissue culture plates using FuGENE6 transfection kit (Promega Inc.). Cell supernatants containing pseudotyped viruses were harvested at 48 hours post transfection and subsequently stored at $-80^{\circ} \mathrm{C}$ until use. The virus infectivity was measured using TZM-bl reporter cells by addition of pseudoviruses

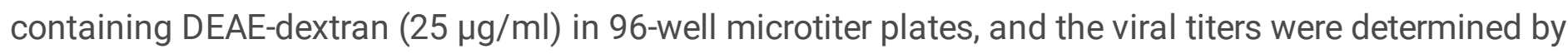
measuring the luciferase activity using Britelite luciferase substrate (PerkinElmer Inc.) with a Victor X2 luminometer (PerkinElmer Inc.).

\section{Neutralization assay.}

Neutralization assays were carried out using TZM-bl cells as described before [37]. Briefly, Envpseudotyped viruses were pre-incubated in 96-well tissue culture plates with various concentrations of bnAbs (IgG) for an hour at $37^{\circ} \mathrm{C}$ in a $\mathrm{CO}_{2}$ incubator under humidified conditions. Subsequently $1 \times 10^{4}$ TZM-bl cells were added to the mixture in the presence of $25 \mu \mathrm{g} / \mathrm{ml}$ DEAE-dextran (Sigma, Inc.). The plates were further incubated for 48 hours. The degree of virus neutralization was assessed by measuring reduction in relative luminescence units (RLU) in a luminometer (Victor X2; PerkinElmer Inc.).

\section{Abbreviations}

HIV: Human Immunodeficiency Virus; bnAb: Broadly neutralizing monoclonal antibodies; [SH]aLRT: Shimodaira-Hasegawa approximate likelihood ratio test; CATNAP: Compile, Analyze and Tally NAb Panels; ARV: Antiretroviral drugs;

\section{Declarations}

\section{Ethics approval and consent to participate.}

The institutional ethical approvals and participant informed consents were obtained prior to collection of biological specimens. 


\section{Consent to publish.}

Not applicable.

\section{Availability of data and materials.}

The dataset(s) supporting the conclusions of this article is (are) included within the article (and its additional file(s). The complete nucleotide sequences of the following envs: 4.2.J45b, 2-7.J1, 2-9.J20, 211.J16, NISA-N20-J10, NISA-N20.J14, NISA-N101.J12, INDO SA NLR 29.J80, INDO SA NLR 29.J11, PG37009v2.eJ9, PG37009v2.eJ38, PG37009v2.eJ58, PG37112v2.J5, PG37112v2.J9, PG37072.J12, PG37072.J16, PG37066.J1, PG37081.J36, PG37087.J39, PG37087.J44, PG37089.J17, PG37089.J20, PG37089.J83, PG37091.J41, PG37080.J6A, PG37080v1.J17, PG37080.J158 are being submitted to the GenBank.

\section{Competing interests.}

The authors declare that they have no competing interests.

\section{Funding.}

The authors wish to acknowledge the funding support from the Wellcome Trust/DBT India Alliance Team Science Grant (IA/TSG/19/1/600019), Science \& Engineering Research Board, Department of Science \& Technology, Government of India (CRG/2019/002939) and Department of Biotechnology, Government of India (BT/PR24520/MED/29/1222/2017). The funders played no role in the design of the study and collection, analysis, interpretation of data and in writing the manuscript.

\section{Authors' contributions.}

JB conceptualized the study; RM, JS, JB planned experiments, RM, NH carried out neutralization assays, JS analyzed sequence data and prepared neutralization heat map analysis, JS, RM, SD, RR, SM, AP contributed gp160 cloning and preparation of pseudoviruses; MT, SS, KGM, AKS, RG helped with providing access to defined patient samples and background information of clinical parameters; DS helped in data analysis; JB, RM, JS wrote the manuscript with help from all the authors.

\section{Acknowledgements.}

We thank Prof Lynn Morris, National Institute for Communicable Diseases, Johannesburg, South Africa for providing us with the plasmids to produce CAP256-VRC26.25 bnAbs used in this study through an 
ongoing collaborative project supported by the Department of Biotechnology, Government of India (BT/PR24520/MED/29/1222/2017). Clinical samples collected under the IAVI Protocol G study was supported by the USAID. We thank the Protocol G study participants registered with YRG Care, Chennai, all of the research staff members at the Protocol G clinical center at YRG Care, Chennai, and all of the IAVI Protocol G team members. IAVI's work was made possible by generous support from many donors, including the Bill \& Melinda Gates Foundation, the Ministry of Foreign Affairs of Denmark, Irish Aid, the Ministry of Finance of Japan, the Ministry of Foreign Affairs of the Netherlands, the Norwegian Agency for Development Cooperation (NORAD), the United Kingdom Department for International Development (DFID), and the United States Agency for International Development (USAID). The full list of IAVI donors is available at www.iavi.org. The contents are the responsibility of the International AIDS Vaccine Initiative and do not necessarily reflect the views of USAID or the United States Government. We thank Prof Gagandeep Kang and Dr Subeer S. Majumdar, Translational Health Science \& Technology Institute for support.

\section{References}

1. Stefic K, Bouvin-Pley M, Braibant M, Barin F: Impact of HIV-1 Diversity on Its Sensitivity to Neutralization. Vaccines (Basel) 2019, 7(3).

2. Sok D, Burton DR: Recent progress in broadly neutralizing antibodies to HIV. Nature immunology 2018, 19(11):1179-1188.

3. Sok D, Le KM, Vadnais M, Saye-Francisco KL, Jardine JG, Torres JL, Berndsen ZT, Kong L, Stanfield R, Ruiz $\mathrm{J}$ et al: Rapid elicitation of broadly neutralizing antibodies to HIV by immunization in cows. Nature 2017, 548(7665):108-111.

4. Walker LM, Huber M, Doores KJ, Falkowska E, Pejchal R, Julien JP, Wang SK, Ramos A, Chan-Hui PY, Moyle $\mathrm{M}$ et al: Broad neutralization coverage of HIV by multiple highly potent antibodies. Nature 2011, 477(7365):466-470.

5. Walker LM, Phogat SK, Chan-Hui PY, Wagner D, Phung P, Goss JL, Wrin T, Simek MD, Fling S, Mitcham JL et al: Broad and potent neutralizing antibodies from an African donor reveal a new HIV-1 vaccine target. Science 2009, 326(5950):285-289.

6. Doria-Rose NA, Bhiman JN, Roark RS, Schramm CA, Gorman J, Chuang GY, Pancera M, Cale EM, Ernandes MJ, Louder MK et al: New Member of the V1V2-Directed CAP256-VRC26 Lineage That Shows Increased Breadth and Exceptional Potency. Journal of virology 2016, 90(1):76-91.

7. Wu X, Yang ZY, Li Y, Hogerkorp CM, Schief WR, Seaman MS, Zhou T, Schmidt SD, Wu L, Xu L et al: Rational design of envelope identifies broadly neutralizing human monoclonal antibodies to HIV-1. Science 2010, 329(5993):856-861.

8. Kumar R, Qureshi H, Deshpande S, Bhattacharya J: Broadly neutralizing antibodies in HIV-1 treatment and prevention. Therapeutic advances in vaccines and immunotherapy 2018, 6(4):61-68.

9. Sok D, van Gils MJ, Pauthner M, Julien JP, Saye-Francisco KL, Hsueh J, Briney B, Lee JH, Le KM, Lee PS et al: Recombinant HIV envelope trimer selects for quaternary-dependent antibodies targeting the 
trimer apex. Proceedings of the National Academy of Sciences of the United States of America 2014, 111(49):17624-17629.

10. Liu Y, Cao W, Sun M, Li T: Broadly neutralizing antibodies for HIV-1: efficacies, challenges and opportunities. Emerging microbes \& infections 2020, 9(1):194-206.

11. Caskey M, Klein F, Lorenzi JC, Seaman MS, West AP, Jr., Buckley N, Kremer G, Nogueira L, Braunschweig M, Scheid JF et al: Viraemia suppressed in HIV-1-infected humans by broadly neutralizing antibody 3BNC117. Nature 2015.

12. Caskey M, Klein F, Nussenzweig MC: Broadly neutralizing anti-HIV-1 monoclonal antibodies in the clinic. Nature medicine 2019, 25(4):547-553.

13. Caskey M, Schoofs T, Gruell H, Settler A, Karagounis T, Kreider EF, Murrell B, Pfeifer N, Nogueira L, Oliveira TY et al: Antibody 10-1074 suppresses viremia in HIV-1-infected individuals. Nature medicine 2017, 23(2):185-191.

14. Mendoza P, Gruell H, Nogueira L, Pai JA, Butler AL, Millard K, Lehmann C, Suarez I, Oliveira TY, Lorenzi JCC et al: Combination therapy with anti-HIV-1 antibodies maintains viral suppression. Nature 2018, 561(7724):479-484.

15. Bar-On Y, Gruell H, Schoofs T, Pai JA, Nogueira L, Butler AL, Millard K, Lehmann C, Suarez I, Oliveira TY et al: Safety and antiviral activity of combination HIV-1 broadly neutralizing antibodies in viremic individuals. Nature medicine 2018, 24(11):1701-1707.

16. Scheid JF, Horwitz JA, Bar-On Y, Kreider EF, Lu CL, Lorenzi JC, Feldmann A, Braunschweig M, Nogueira L, Oliveira T et al: HIV-1 antibody 3BNC117 suppresses viral rebound in humans during treatment interruption. Nature 2016, 535(7613):556-560.

17. Binley JM, T. Wrin, B. Korber, M. B. Zwick, M. Wang, C. Chappey, G. Stiegler, R. Kunert, S. Zolla-Pazner, H. Katinger, C. J. Petropoulos, and, Burton. DR: Comprehensive cross-clade neutralization analysis of a panel of anti-human immunodeficiency virus type 1 monoclonal antibodies. Journal of virology 2004, 78:13232-13252.

18. Bures R, Morris L, Williamson C, Ramjee G, Deers M, Fiscus SA, Abdool-Karim S, Montefiori DC: Regional clustering of shared neutralization determinants on primary isolates of clade $\mathrm{C}$ human immunodeficiency virus type 1 from South Africa. Journal of virology 2002, 76(5):2233-2244.

19. Hraber P, Korber BT, Lapedes AS, Bailer RT, Seaman MS, Gao H, Greene KM, McCutchan F, Williamson C, Kim JH et al: Impact of clade, geography, and age of the epidemic on HIV-1 neutralization by antibodies. Journal of virology 2014, 88(21):12623-12643.

20. Kulkarni SS, Lapedes A, Tang H, Gnanakaran S, Daniels MG, Zhang M, Bhattacharya T, Li M, Polonis VR, McCutchan FE et al: Highly complex neutralization determinants on a monophyletic lineage of newly transmitted subtype C HIV-1 Env clones from India. Virology 2009, 385(2):505-520.

21. Seaman MS, Janes H, Hawkins N, Grandpre LE, Devoy C, Giri A, Coffey RT, Harris L, Wood B, Daniels MG et al: Tiered categorization of a diverse panel of HIV-1 Env pseudoviruses for assessment of neutralizing antibodies. Journal of virology 2010, 84(3):1439-1452. 
22. Burnie J, Guzzo C: The Incorporation of Host Proteins into the External HIV-1 Envelope. Viruses 2019, 11(1).

23. Rusert P, Kouyos RD, Kadelka C, Ebner H, Schanz M, Huber M, Braun DL, Hoze N, Scherrer A, Magnus $\mathrm{C}$ et al: Determinants of HIV-1 broadly neutralizing antibody induction. Nature medicine 2016, 22(11):1260-1267.

24. Novitsky V, Smith UR, Gilbert P, McLane MF, Chigwedere P, Williamson C, Ndung'u T, Klein I, Chang SY, Peter $\mathrm{T}$ et al: Human immunodeficiency virus type 1 subtype $\mathrm{C}$ molecular phylogeny: consensus sequence for an AIDS vaccine design? Journal of virology 2002, 76(11):5435-5451.

25. Wagh K, Bhattacharya T, Williamson C, Robles A, Bayne M, Garrity J, Rist M, Rademeyer C, Yoon H, Lapedes A et al: Optimal Combinations of Broadly Neutralizing Antibodies for Prevention and Treatment of HIV-1 Clade C Infection. PLoS pathogens 2016, 12(3):e1005520.

26. Lorenzi JCC, Mendoza P, Cohen YZ, Nogueira L, Lavine C, Sapiente J, Wiatr M, Mugo NR, Mujugira A, Delany $S$ et al: Neutralizing Activity of Broadly Neutralizing anti-HIV-1 Antibodies against Primary African Isolates. Journal of virology 2020.

27. Bai H, Li Y, Michael NL, Robb ML, Rolland M: The breadth of HIV-1 neutralizing antibodies depends on the conservation of key sites in their epitopes. PLoS computational biology 2019, 15(6): 1007056.

28. Rademeyer C, Korber B, Seaman MS, Giorgi EE, Thebus R, Robles A, Sheward DJ, Wagh K, Garrity J, Carey BR et al: Features of Recently Transmitted HIV-1 Clade C Viruses that Impact Antibody Recognition: Implications for Active and Passive Immunization. PLoS pathogens 2016, 12(7):e1005742.

29. Crowell TA, Colby DJ, Pinyakorn S, Sacdalan C, Pagliuzza A, Intasan J, Benjapornpong K, Tangnaree $\mathrm{K}$, Chomchey N, Kroon E et al: Safety and efficacy of VRC01 broadly neutralising antibodies in adults with acutely treated HIV (RV397): a phase 2, randomised, double-blind, placebo-controlled trial. The lancet HIV2019, 6(5):e297-e306.

30. Reeves DB, Huang Y, Duke ER, Mayer BT, Cardozo-Ojeda EF, Boshier FA, Swan DA, Rolland M, Robb $\mathrm{ML}$, Mascola JR et al: Mathematical modeling to reveal breakthrough mechanisms in the HIV Antibody Mediated Prevention (AMP) trials. PLoS computational biology 2020, 16(2):e1007626.

31. Mahomed S, Garrett N, Karim QA, Zuma NY, Capparelli E, Baxter C, Gengiah T, Archary D, Samsunder $\mathrm{N}$, Rose ND et al: Assessing the safety and pharmacokinetics of the anti-HIV monoclonal antibody CAP256V2LS alone and in combination with VRC07-523LS and PGT121 in South African women: study protocol for the first-in-human CAPRISA 012B phase I clinical trial. BMJ Open 2020, 10(11):e042247.

32. Gharu L, Ringe R, Bhattacharya J: HIV-1 clade C envelopes obtained from late stage symptomatic Indian patients varied in their ability towards relative CD4 usages and sensitivity to CCR5 antagonist TAK-779. Virus research 2011, 158:216-224.

33. Gharu L, Ringe R, Bhattacharya J: Evidence of extended alternate coreceptor usage by HIV-1 clade C envelope obtained from an Indian patient. Virus research 2012, 163(1):410-414. 
34. Gharu L, Ringe R, Pandey S, Paranjape R, Bhattacharya J: HIV-1 clade C env clones obtained from an Indian patient exhibiting expanded coreceptor tropism are presented with naturally occurring unusual amino acid substitutions in V3 loop. Virus research 2009, 144(1-2):306-314.

35. Ringe R, Thakar M, Bhattacharya J: Variations in autologous neutralization and CD4 dependence of b12 resistant HIV-1 clade $C$ env clones obtained at different time points from antiretroviral naive Indian patients with recent infection. Retrovirology 2010, 7(1):76.

36. Mukhopadhyay S, Ringe R, Patil A, Paranjape R, Bhattacharya J: Characterization of Circulating HIV Type 1 env Genes in Plasma of Two Antiretroviral-Naive Slow Progressing Patients with Broad Neutralizing Antibody Response with Evidence of Recombination. AIDS research and human retroviruses 2012, 28(7):739-745.

37. Patil S, Kumar R, Deshpande S, Samal S, Shrivastava T, Boliar S, Bansal M, Chaudhary NK, Srikrishnan AK, Murugavel KG et al: Conformational Epitope-Specific Broadly Neutralizing Plasma Antibodies Obtained from an HIV-1 Clade C-Infected Elite Neutralizer Mediate Autologous Virus Escape through Mutations in the V1 Loop. Journal of virology 2016, 90(7):3446-3457.

38. Deshpande S, Patil S, Kumar R, Hermanus T, Murugavel KG, Srikrishnan AK, Solomon S, Morris L, Bhattacharya J: HIV-1 clade C escapes broadly neutralizing autologous antibodies with N332 glycan specificity by distinct mechanisms. Retrovirology 2016, 13(1):60.

39. Sutar J, Deshpande S, Mullick R, Hingankar N, Patel V, Bhattacharya J: Geospatial HIV-1 subtype C gp120 sequence diversity and its predicted impact on broadly neutralizing antibody sensitivity. BioRxiv 2020, October 31, 2020.(doi https://doi.org/10.1101/2020.09.09.289132).

40. Krumm SA, Mohammed H, Le KM, Crispin M, Wrin T, Poignard P, Burton DR, Doores KJ: Mechanisms of escape from the PGT128 family of anti-HIV broadly neutralizing antibodies. Retrovirology 2016, 13(1):8.

41. Ringe R, Phogat S, Bhattacharya J: Subtle alteration of residues including N-linked glycans in V2 loop modulate HIV-1 neutralization by PG9 and PG16 monoclonal antibodies. Virology 2012, 426(1):34-41.

42. Kist NC, Lambert B, Campbell S, Katzourakis A, Lunn D, Lemey P, Iversen AKN: HIV-1 p24Gag adaptation to modern and archaic HLA-allele frequency differences in ethnic groups contributes to viral subtype diversification. Virus Evol 2020, 6(2):veaa085.

43. Hall T: BioEdit: a user-friendly biological sequence alignment editor and analysis program for Windows 95/98/NT. . Nucleic Acids Symp 1999(https://doi.org/citeulike-article-id:691774).

44. Rice P, Longden I, Bleasby A: EMBOSS: the European Molecular Biology Open Software Suite. Trends Genet 2000, 16(6):276-277.

45. Zhang M, Gaschen B, Blay W, Foley B, Haigwood N, Kuiken C, Korber B: Tracking global patterns of Nlinked glycosylation site variation in highly variable viral glycoproteins: HIV, SIV, and HCV envelopes and influenza hemagglutinin. Glycobiology 2004, 14(12):1229-1246.

46. Nguyen LT, Schmidt HA, von Haeseler A, Minh BQ: IQ-TREE: a fast and effective stochastic algorithm for estimating maximum-likelihood phylogenies. Molecular biology and evolution 2015, 32(1):268- 
274.

47. Yu G, Lam TT, Zhu H, Guan Y: Two Methods for Mapping and Visualizing Associated Data on

Phylogeny Using Ggtree. Molecular biology and evolution 2018, 35(12):3041-3043.

48. Yu G, Smith D, Zhu H, Guan Y, Lam TT: ggtree: an R package for visualization and annotation of

phylogenetic trees with their covariates and other associated data. Methods Ecol Evol 2017, 8:28-36.

49. Team R: RStudio: Integrated Development for R. 2015.

50. Team RC: R: A language and environment for statistical computing. 2018.

\section{Tables}

Due to technical limitations, table 1 is only available as a download in the Supplemental Files section.

\section{Figures}
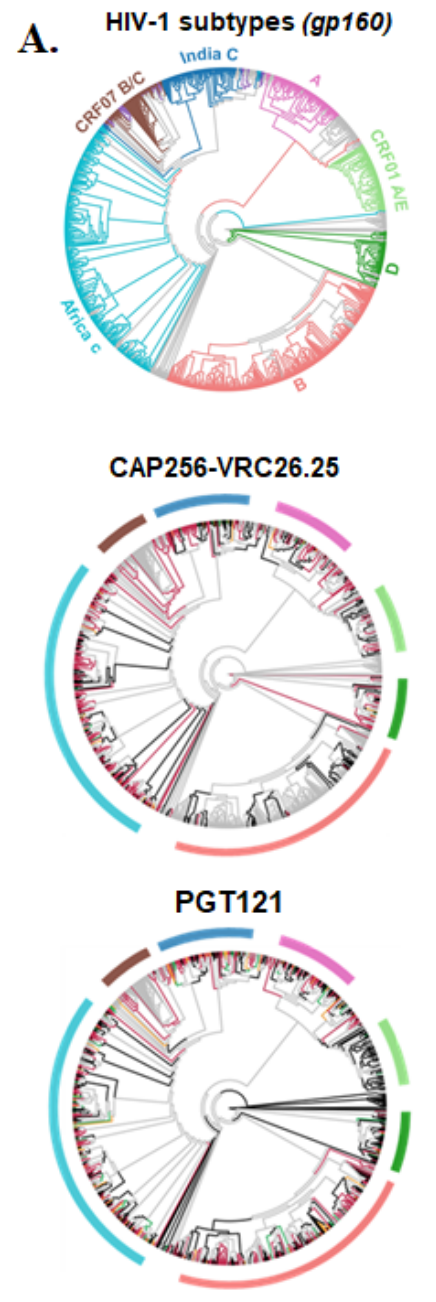

B.
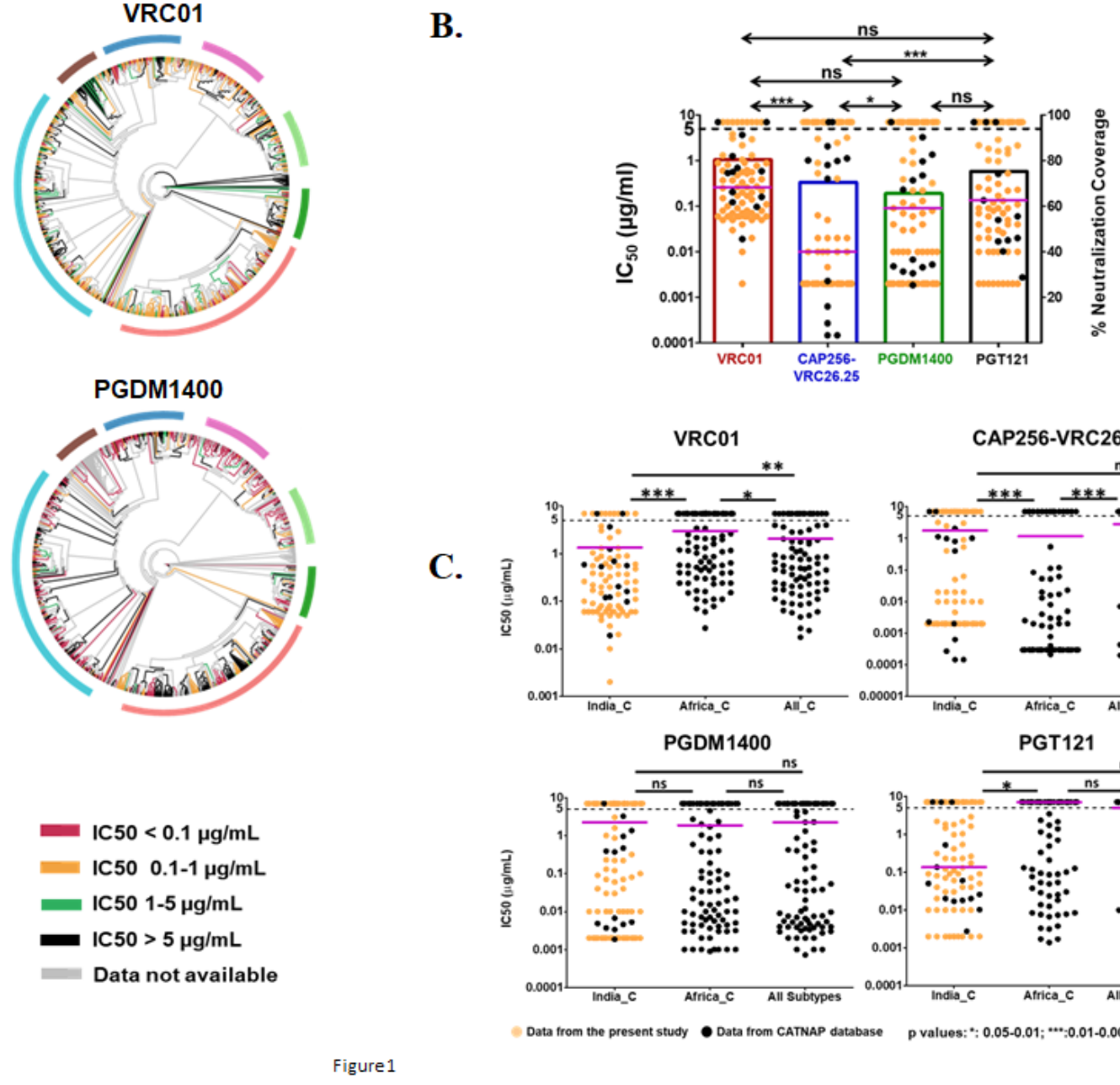
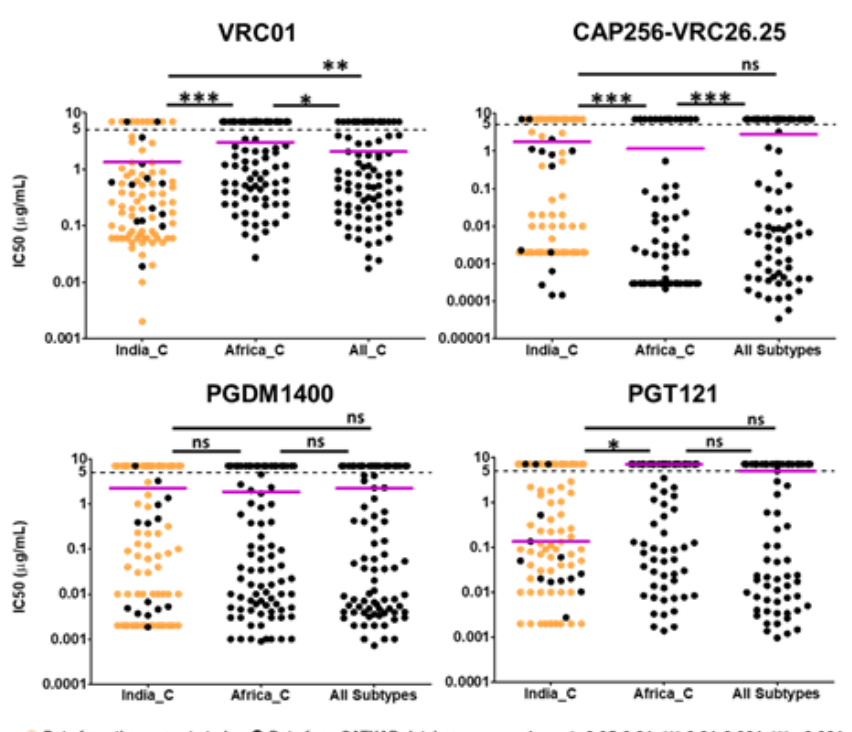

Data from the present study $\bullet$ Data from CATNAP database p values: ${ }^{*}: 0.05-0.01 ; \cdots ; 0.01-0.001, \cdots ;<0.001$

Figure 1

Association of phylogenetic relatedness of env and their responses when expressed as pseudovirus to the four bnAbs A. A phylogenetic tree was constructed for gp160 amino acid sequences of viral clones 
reported in the present study along with those reported in the CATNAP database (Total $N=1020$ ). The terminal branches of the tree were color-coded based on the subtype as depicted in the color legend. Four heatmaps based on their responses to bnAbs PGDM1400, PGT121, VRC01 and CAP256-VRC26.25 were overlayed on the phylogenetic tree to assess phylogenetic clustering of their IC50 values. IC50 value of $5 \mathrm{ug} / \mathrm{mL}$ were considered as neutralization sensitivity threshold. B. Neutralization potency (scatter plot against left $Y$ axis) was plotted for pseudoviruses expressing 98 subtype $C$ envs (71 from the present study) from India against the four bnAbs. Pink lines indicate median IC50 values. Orange dots represent data generated in the present study while the black dots indicate data retrieved from the CATNAP database. Potency against all pairs of bnAbs were compared using Mann-Whitney test. $p$ values were

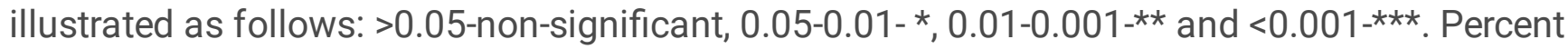
neutralization coverage of all the bnAbs were plotted as a bar chart against the right $Y$ axis. $C$. Year matched randomly selected equal number viral clone datasets were retrieved from CATNAP database and their IC50 responses to 4 bnAbs (PGT121, PGDM1400, VRC01 and CAP256-VRC26.25 were compared between Subtype $C$ from India, Subtype $C$ pan Africa and Other subtypes (All except C). IC50 value of $5 \mathrm{ug} / \mathrm{mL}$ was considered as a threshold of neutralization sensitivity. Statistical comparisons were made between each pair for every antibody using Mann-Whitney test.
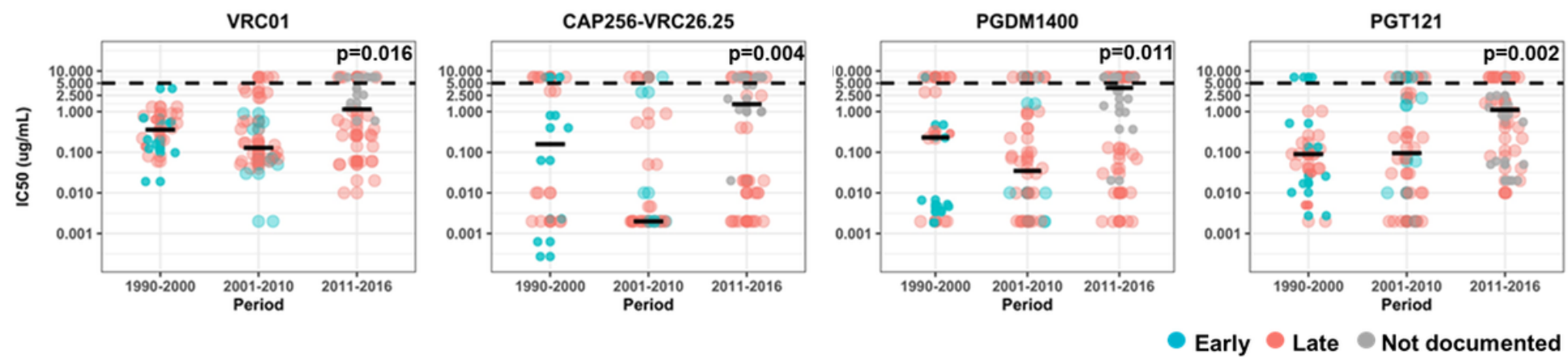

Figure 2

\section{Figure 2}

Comparison of trend in bnAb sensitivity across time against VRC01, CAP256-VRC26.25, PGDM1400 and PGT121. Scatter plots of IC50 values for clones reported in the CATNAP database were grouped as 19902000, 2001-2010 and 2011-2016 for viruses from India along with those reported in the present study (indicated by larger dots). Data points were color-coded based on the disease stage at sampling of the respective viruses. IC50 value of $5 \mathrm{ug} / \mathrm{mL}$ was considered as a threshold of neutralization sensitivity. Statistical assessment of increase in IC50 values was performed with Jonckheere-Terpstra test (JTT). 
NARI IVC-2

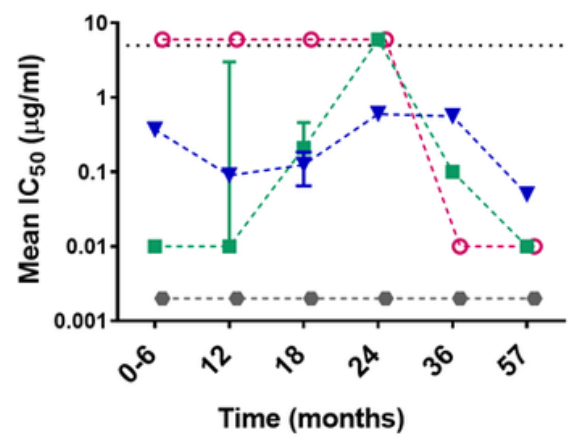

NARI IVC-3

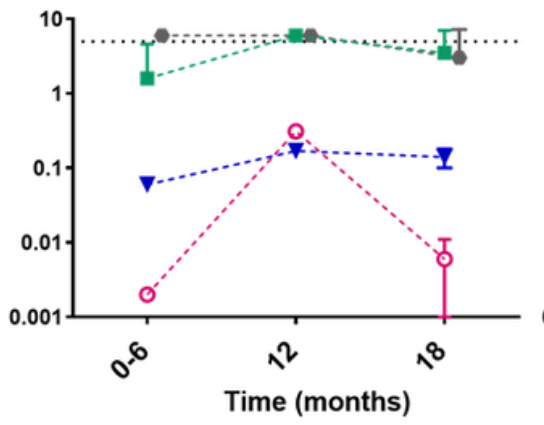

NARI IVC-11

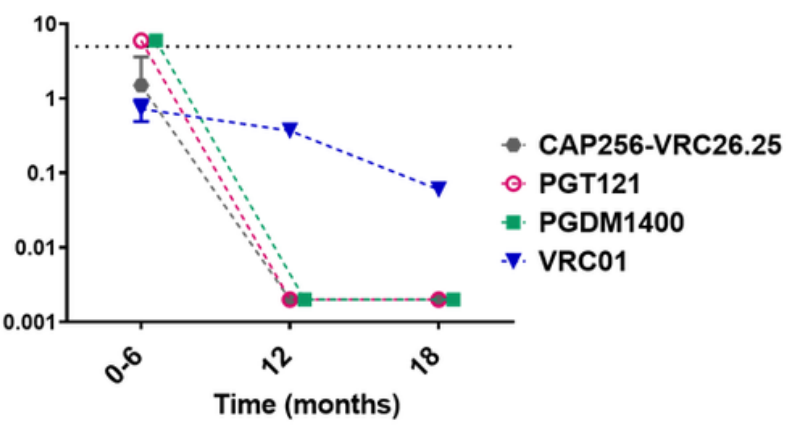

Figure 3

Figure 3

Progression of bnAb responses of pseudoviruses expressing envs obtained from longitudinally followed up individuals. Mean IC50 values against bnAbs VRC01, CAP256-VRC26.25, PGDM1400 and PGT121 and were plotted for viral clones prepared from longitudinal collected samples from three HIV-1 subtype $\mathrm{C}$ infected individuals (NARI IVC-2, NARI IVC-3 and NARI IVC-11). IC50 value of $5 \mu \mathrm{g} / \mathrm{ml}$ were considered as a threshold of neutralization sensitivity.

A
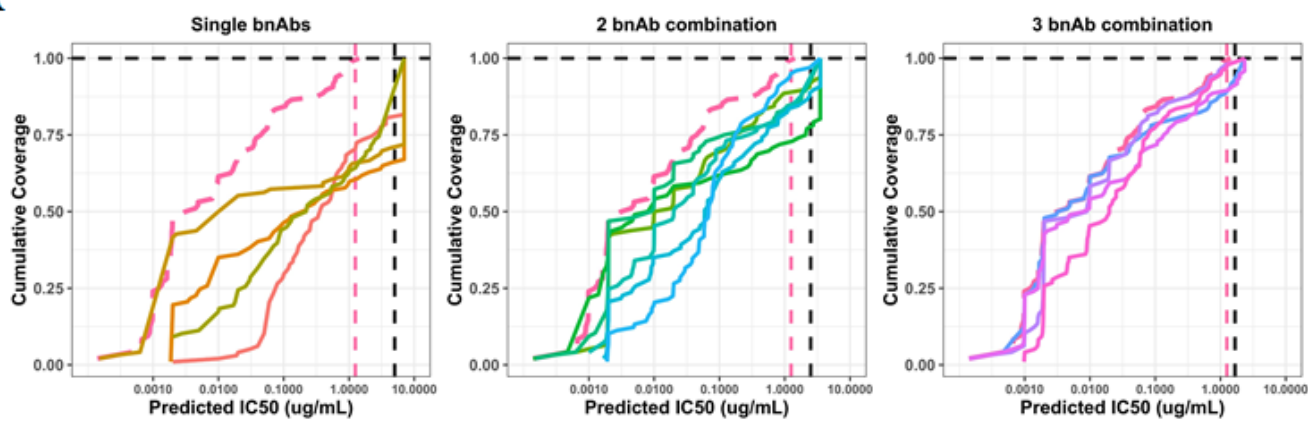

B

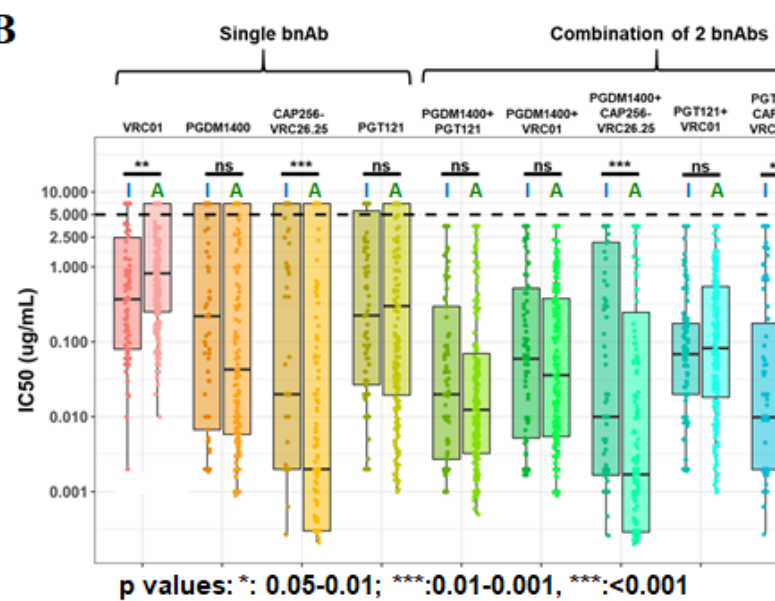

Single bnAbs

VRC01

PGDM1400

PGT121

CAP256-VRC26.25

Combination of 2 bnAbs

PGDM1400 + PGT121

PGDM1400 + VRC01

PGDM1400 + CAP256VRC26.25

PGT121 + VRC01

PGT121 + CAP256-VRC26.25

VRC01 + CAP256-VRC26.25

Combination of 3 bnAbs

PGDM1400 + PGT121 + VRC01

PGDM1400 + PGT121 +

CAP256-VRC26.25

PGDM1400 + VRC01 + CAP256-VRC26.25

PGT121 + VRC01 + CAP256VRC26.25

Combination of 4 bnAbs

PGDM1400 + PGT121 + VRC01

+ CAP256-VRC26.25

Figure 4 
Predictive analysis of effect of bnAb combinations on neutralization breadth and potency of HIV-1 Indian clade C. A. Cumulative coverage of the virus panel (a fraction between $0 \& 1$ ) of 98 clones reported in the present study and CATNAP database from India at various IC50 values for single mAbs and/or mAb combinations with a target $A b$ concentration of $5 \mu \mathrm{g} / \mathrm{ml}$. Dashed curve in each of the plots indicates 4 bnAb combination. All other combinations are depicted according to the given color codes. Vertical dashed lines in each plot indicate the expected final $A b$ concentration of four bnAb combination (pink line) and combination under assessment (black line). B. Predicted geometric IC50 values against each bnAb/combination based on experimental IC50 values for viruses from India (N-98, left boxplot in each group) vs Viruses from Africa retrieved from CATNAP ( $N=250$, right boxplot in each group). Black lines in each plot indicate Median IC50 values. Statistical comparison within each group was performed with Mann-Whitney test.

\section{Supplementary Files}

This is a list of supplementary files associated with this preprint. Click to download.

- FIG.S1.tif

- TABLES1FINAL.docx

- TABLES2Final.docx

- Table1.docx 